\title{
Cognitive Abilities in First-Degree Relatives of Individuals with Bipolar Disorder
}

Daniela Calafiore ${ }^{1}$, Susan L. Rossell ${ }^{1,2,3}$ and Tamsyn E. Van Rheenen ${ }^{1,3,4^{*}}$

Affiliations:

${ }^{1}$ Centre for Mental Health, Faculty of Health, Arts and Design, School of Health Sciences, Swinburne University, Melbourne, Australia

${ }^{2}$ Cognitive Neuropsychiatry Laboratory, Monash Alfred Psychiatry Research Centre

(MAPrc), The Alfred Hospital and Central Clinical School, Monash University, Melbourne, Australia.

${ }^{3}$ Department of Psychiatry, St Vincent's Hospital, Melbourne, Australia

${ }^{4}$ Melbourne Neuropsychiatry Centre, Department of Psychiatry, University of Melbourne, Melbourne, Australia

*Address correspondence to:

Dr Tamsyn Van Rheenen

Melbourne Neuropsychiatry Centre

Level 3, Alan Gilbert Building

161 Barry St, Carlton, Vic 3053, Australia

tamsyn.van@unimelb.edu.au 


\section{Abstract}

Background: Although the study of cognition in first degree relatives (FDRs) is not new, findings in this group are still somewhat inconsistent and much of the research examining FDR populations include individuals under the age of 25 , who are arguably still at significant risk to go on to develop BD. The present study aimed to establish the value of cognitive performance as a genuine endophenotypic marker of familial risk for bipolar disorder (BD), by examining cognition in FDRs aged 25 years or older.

Methods: The current study compared the cognitive performance of 27 unaffected FDRs to 47 healthy controls (HCs) and 28 BD patients using the MATRICS Consensus Cognitive Battery (MCCB).

Results: Results indicated that FDRs had impaired verbal learning performance, as well as selective impairments on a measure of speed of processing; and a measure of spatial working memory compared to HC.

Limitations: Limitations relate to the potential insensitivity of some of the tests in the MCCB for detecting cognitive deficits that have been previously noted in $\mathrm{BD}$ and FDR samples using other batteries.

Conclusions: Findings from this study implicate verbal learning, processing speed and working memory performance as promising candidate endophenotypes of familial risk for BD.

Key Words: Cognition; Bipolar disorder; Endophenotype; Verbal learning; Executive functioning; Problem-solving; Heritability; Familial risk 
Cognitive deficits are well documented in bipolar disorder (BD), particularly in the domains of sustained attention, verbal learning and executive functioning (Balanza-Martinez et al., 2008; Bora et al., 2009; Clark and Goodwin, 2004; Douglas and Van Rheenen, 2016; Martinez-Aran et al., 2000; Robinson and Ferrier, 2006; Russo et al., In press; Van Rheenen et al., 2017; Van Rheenen et al., 2016; Van Rheenen and Rossell, 2014a). These deficits are evident during symptomatic episodes, but converging evidence suggests that patients continue to experience persistent cognitive impairment, albeit to a lesser degree, across a range of tasks during symptom remission (Antila et al., 2007b; Arts et al., 2008; Bearden et al., 2010; Bora et al., 2009; Kurtz and Gerraty, 2009; Mann-Wrobel et al., 2011; MartinezAran et al., 2004; Robinson et al., 2006; Thompson et al., 2005; Torres et al., 2007). The enduring nature of these deficits suggests that they represent trait rather than state abnormalities of the disorder.

Cognitive impairments observed in unaffected first-degree relatives (FDRs) may serve as endophenotypic markers for BD, particularly if FDRs exhibit an intermediate pattern of performance when compared to BD patients and healthy controls (HC). Investigating cognitive function in healthy individuals with high familial risk of BD, such as unaffected FDRs, can avoid many of the confounds of BD studies, such as medication use, the presence of mood symptoms (either clinical or sub-clinical), and the possibility that patients have endured lasting neuroanatomical changes as a result of the illness (Antila et al., 2007b; Hellvin et al., 2012). As such, research attention focussed on FDRs offer a unique means by which to explore the heritability of cognitive impairments in BD.

Although the study of cognition in relatives of individuals with $\mathrm{BD}$ is not new, findings in this group are still somewhat inconsistent (Balanza-Martinez et al., 2008; Cardenas et al., 2016; Hasler et al., 2006). For example, current work suggests that endophenotypic markers of genetic vulnerability for BD may be represented by deficits in 
verbal memory (Arts et al., 2008; Balanza-Martinez et al., 2008; Cardenas et al., 2016; Kieseppä et al., 2005; Kulkarni et al., 2010; McIntosh et al., 2005) and selective deficits in aspects of executive function and sustained attention (Arts et al., 2008; Bauer et al., 2016; Bora et al., 2008; Clark et al., 2005b; Ferrier et al., 2004a; Glahn et al., 2004; Nehra et al., 2006; Trivedi et al., 2008; Zalla et al., 2004), since FDRs have shown impairments on these domains relative to HCs. Speed of processing and verbal working memory deficits also seem to be related to genetic risk for BD, yet results on these domains are mixed, with some studies evidencing deficits in FDRs and others not (Antila et al., 2007b; Cardenas et al., 2016; Daban et al., 2012; Nehra et al., 2006; Pierson et al., 2000).

Discrepant findings in the literature suggest that the cognitive profile of relatives of BD patients is still unclear and requires replication in well-defined samples; this includes samples in which the modal age of BD onset has been taken into account. Our review of the literature indicates that much of the research examining FDR populations include individuals between the ages of 18 to 25 . As BD typically develops during late adolescence and early adulthood, these individuals are arguably still at significant risk to go on to develop BD (Baldessarini et al., 2012). Thus, assessment of FDRs in this age-bracket blurs understandings of the extent to which cognitive deficits represent true familial risk as opposed to premorbid processes occurring prior to illness onset. The current study aimed to overcome this limitation by comparing the cognitive performance of unaffected FDRs aged 25 years or older, to HCs and BD patients across a battery of cognitive tasks. Hence, we aimed to establish the value of cognitive performance as a genuine endophenotypic marker of familial risk for BD. On the basis of previous literature we predicted that unaffected FDRs of individuals with BD would show selective cognitive dysfunction on measures of verbal memory, executive function, and speed of processing when compared to HCs, and that the magnitude of dysfunction would be intermediate to that of BD patients and HCs. 


\section{Method}

This study was approved by the relevant Human Ethics Review Boards and abided by the Declaration of Helsinki. Written informed consent was obtained from each participant before the study commenced.

\section{Participants}

The FDR sample comprised 27 individuals over the age of 25 , with a first-degree biological sibling or parent with a diagnosis of BD (I or II) and no current or past history of psychiatric disorder. The clinical sample comprised 28 patients with a DSM-IV-TR diagnosis of BD-I. These BD participants were drawn from a pre-existing database and as such, the neurocognitive performance of parts of this sample has previously been reported (Van Rheenen et al., 2017; Van Rheenen et al., 2016; Van Rheenen et al., 2014; Van Rheenen and Rossell, 2014a). FDRs were unrelated to individuals in the BD sample in this study to circumvent the effect of shared environmental influences on cognitive performance. A sample of $47 \mathrm{HC}$ participants was recruited for comparison purposes. The Mini International Neuropsychiatric Interview (MINI) (Sheehan et al., 1998) was used to screen all participants for psychiatric disorder. Current mood symptomology was assessed using the Young Mania Rating Scale (YMRS) (Young et al., 1978) and the Montgomery Asberg Depression Rating Scale (MADRS) (Montgomery and Asberg, 1979).

Exclusion criteria included: i) difficulties with spoken English, ii) a history of traumatic brain injury, iii) hearing or visual impairments, iv) neurological or degenerative illness, v) alcohol or substance abuse/dependence in the past 3 months, vi) pregnancy, vii) an estimated IQ of less than 75 on the Wechsler Test of Adult Reading (WTAR) (Wechsler, 2001), viii) a history of psychotropic medication use such as antidepressants, antipsychotics, 
benzodiazepines and mood stabilizers (FDRs and HCs), xi) a family history of mood or psychiatric disorder (HCs only) or $\mathrm{x}$ ) within the age range of 18-65 (HC or $\mathrm{BD}$ ) or 25-65 years (FDRs). Participants were recruited using general advertisements as well as online websites and social media. Participants were reimbursed for their participation.

\section{Materials}

The MATRICS Consensus Cognitive Battery (MCCB)(Nuechterlein and Green, 2006) was used as a measure of cognitive functioning, assessing the domains of attention/vigilance (Continuous Performance Test- identical pairs[CPT-IP]), speed of processing (Tail Making Test -A[TMT-A], Brief Assessment of Cognition in Schizophrenia Symbol Coding [BACS-SC], Animal Naming), working memory (Wechsler Memory Scale Spatial Span[WMS-R], Letter-Number Sequencing[LNS]), visual learning (Brief Visual Memory Test-Revised [BVMT-R]) verbal learning (Hopkins Verbal Learning Test Revised [HVLT-R]), reasoning/problem solving (Neuropsychological Assessment Battery [NAB]Mazes) and social cognition (Mayer-Salovey-Caruso Emotional Intelligence Test [MSCEIT]). The MCCB has been used in BD previously (Burdick et al., 2011a; Van Rheenen and Rossell, 2014a) and shows good test-retest reliability. Supplementary Table 1 provides a summary of the MCCB domain measures and subtests.

\section{Statistical Analysis}

Chi square analyses and analysis of variance (ANOVA) with follow-up Least Significant Difference (LSD) t-tests were used to compare group differences on key demographic and clinical characteristics. Raw scores on the neuropsychological tests were transformed to standard equivalents $(z$-scores with a mean $=0$ and $\mathrm{SD}=1)$ based on the 
means and standard deviations of the HC group. The TMT-A had a different metric to the other tests and was reversed to be consistent with the other measures, so that higher test scores represented better performance. On domains that comprised more than one test measure (i.e., speed of processing and working memory), a composite score was created from the summed $z$ scores; this was then re-standardised. Standardised domain and subtest scores were entered into two separate multivariate analyses of covariance analyses (MANCOVA), with post-hoc LSD correction to assess group differences; age and gender were added into the analyses as covariates $a$-priori given that they are known to be associated with cognitive performance (Lezak et al., 2004; Strauss et al., 2006; Tombaugh et al., 1999). The domains of the MCCB were entered as fixed factors.

Exploratory bivariate correlations were conducted to examine the association of scores of the symptom rating scales (YMRS and MADRS) with cognitive test performance in the BD and FDR group separately. Conservative $\alpha=.01$ was used for all correlational and post-hoc tests to account for multiple testing.

\section{Results}

\section{Demographics}

Table 1 displays the demographic and clinical descriptives for the sample. As expected, the BD group differed significantly from HCs and FDRs on both the MADRS and YMRS. No significant differences were found between groups on age, premorbid IQ or gender distribution. 


\section{Cognitive Performance}

There was a significant omnibus group effect for cognitive performance (Pillai's Trace $\left.=.34, F(14,178)=2.64, p=.002, \eta^{2}=.17\right)$, with BD patients showing the greatest impairment overall $(M=31.15, \mathrm{SD}=7.14)$, and FDRs $(M=31.45, \mathrm{SD}=5.39)$ performing less accurately than $\mathrm{HCs}(M=32.98, \mathrm{SD}=5.92)$. Inspection of each domain showed no group differences for speed of processing $(F(2,94)=.79, p=.460)$, attention/vigilance $(F(2$, $94)=.17, p=.840)$, or social cognition $(F(2,94)=.77, p=.470)$. Significant group differences were evident for verbal learning $(F(2,94)=4.29, p=.016)$, visual learning $(F(2$, $94)=3.99, p=.02)$, working memory $(F(2,94)=5.76, p=.004)$ and reasoning $/$ problem solving $(F(2,94)=4.22, p=.018)$. Follow up pairwise comparisons revealed that FDRs had worse verbal learning performance than $\mathrm{HCs}$ (mean difference $=.78, \mathrm{SE}=.28, p=.006$ ). There was a trend for a difference in verbal learning between HCs and BD patients (mean difference $=.52, \mathrm{SE}=.28, p=.065)$, but no difference in performance between BD patients and FDRs (mean difference $=.27, \mathrm{SE}=.31, p=.39$ ). The $\mathrm{BD}$ group performed worse than $\mathrm{HCs}$ on the visual learning domain (mean difference $=.72, \mathrm{SE}=.25, p=.006$ ), but no significant differences were found between BD patients and FDRs (mean difference $=.43, \mathrm{SE}$ $=.28, p=.13$ ) or HCs and FDRs (mean difference $=.29, \mathrm{SE}=.26, p=.27$ ). Both BD patients $($ mean difference $=.70, \mathrm{SE}=.23, p=.003)$ and FDRs (mean difference $=-.61, \mathrm{SE}=.23, p=$ .011) performed worse than HCs on the working memory domain, but there were no significant differences between them (mean difference $=.09, \mathrm{SE}=.26, p=.72$ ). However, $\mathrm{BD}$ patients performed worse than FDRs (mean difference $=.67, \mathrm{SE}=.24, p=.007$ ) but not HCs (mean difference $=.15, \mathrm{SE}=.22, p=.49)$ on the problem-solving domain; there was a trend for better performance in the FDRs compared to HCs (mean difference $=.52, \mathrm{SE}=.22$, 
$p=.022$ ). Figure 1a presents a graphical depiction of $Z$ scores on each domain for each group, while Table 2 presents the effect size differences (Cohen's d) between groups.

A second MANCOVA conducted on the individual sub-tests of the domains with more than one subtest indicated a significant omnibus effect (Pillai's Trace $=.28, F(10,184)$ $\left.=3.004, p=.002, \eta^{2}=.14\right)$. Group differences were evident on the BACS-SC $(F(2,95)=$ $5.18, p=.007)$ and the WMS-SS $(F(2,95)=8.59, p=.001)$, but not the $\operatorname{LNS}(F(2,95)=$ $2.06, p=.13)$, Animal Naming $(F(2,95)=1.60, p=.21)$ or the TMT-A $(F(2,95)=2.35, p=$ .10). Follow up pairwise comparisons indicated that both $\mathrm{BD}$ (mean difference $=.57, \mathrm{SE}=$ $.21, p=.008)$ and FDR (mean difference $=.55, \mathrm{SE}=.21, p=.01)$ groups performed significantly worse than HCs on the BACS-SC, but did not differ significantly from each other $($ mean difference $=.02, \mathrm{SE}=.23, p=.93)$. Similarly, both $\mathrm{BD}($ mean difference $=1.01$, $\mathrm{SE}=.26, p=.001)$ and FDRs (mean difference $=.77, \mathrm{SE}=.26, p=.005)$ performed worse on the WMS-SS than HCs, but did not significantly differ from each other (mean difference = $.24, \mathrm{SE}=.29, p=.41)$. Figure $1 \mathrm{~b}$ presents a graphical depiction of $\mathrm{Z}$ scores on each domain for each group, while Table 2 includes the effect size differences (Cohen's d) between groups for each subtest.

\section{Correlations}

Cognitive performance on any of the subtest scores or domain scores did not correlate with depression (MADRS) or mania severity ratings (YMRS) in either the BD (lowest p MADRS $=.12, \mathrm{YMRS}=.09$ )or FDR (lowest $\mathrm{p}$ MADRS $=.10, \mathrm{YMRS}=.07)$ groups.

\section{Discussion}

In the interests of establishing potential endophenotypic markers of $\mathrm{BD}$, the current study explored cognitive abilities in unaffected FDRs of BD patients across a battery of 
cognitive tasks. FDRs showed significant impairment on the verbal learning domain when compared to HCs, which is partially consistent with previous literature that has indicated that FDRs exhibit cognitive impairment on measures of verbal memory as well as executive function and speed of processing when compared to HCs (Arts et al., 2008; Balanza-Martinez et al., 2008; Bora et al., 2009; Ferrier et al., 2004a; Hasler et al., 2006; Kieseppä et al., 2005; McIntosh et al., 2005). This FDR-HC impairment was slightly greater in magnitude (albeit not significant) than that exhibited by BD patients, whose verbal learning only differed from HCs at trend level. Additionally, in our sample although not significant, FDRs were qualitatively intermediate in their visual learning performance compared to BD and HCs, with BD patients performing worse than HCs.

Impairment was also noted between FDRs and HCs on the working memory domain; secondary analysis on the individual tests contained within the composite domain score indicated that the primary driver of this effect was the WMS-SS and not the LNS. Deficits on the WMS-SS are consistent with those reported by Ferrier and colleagues (Ferrier et al., 2004b), who found FDRs were impaired on measures of visual working memory. The current lack of significant differences on the verbal working memory task are in line with those of Antila and colleagues (Antila et al., 2007a), but contrast three other studies that found deficits in FDRs (Bora et al., 2008; Ferrier et al., 2004a; Gourovitch et al., 1999).

No significant impairment was found in FDRs on measures of attention/vigilance, problem solving, social cognition or speed of processing when compared to HCs. Since there is some evidence to suggest that sustained attention impairments in BD samples may be state dependent, the lack of attention deficits in FDRs is perhaps not surprising. It is certainly consistent with several previous studies that have reported that FDRs have unimpaired sustained attention compared to HCs using a range of measures (Balanza-Martinez et al., 2008; Clark et al., 2005a; Kieseppä et al., 2005; Kulkarni et al., 2010; Sobczak et al., 2003). 
Closer examination of the individual tasks contributing to the speed of processing domain revealed significant group differences on the BACS-SC, with FDRs demonstrating performance decrements when compared to the HCs. As the need to hold and manipulate visual information mentally is an important component of this task (Joy et al., 2004), it is plausible that the spatial working memory deficit observed in this sample may have contributed to the impairment. Consistent with other findings, no significant differences were found between groups on the TMT-A (Antila et al., 2007b; Balanza-Martinez et al., 2008; Bora et al., 2008; Christensen et al., 2006; Ferrier et al., 2004a; Gourovitch et al., 1999; Klimes-Dougan et al., 2006; Kremen et al., 1998; Zalla et al., 2004) or on the animal naming verbal fluency test (Zalla et al., 2004).

In comparing the FDR and BD groups, significant differences were only found on the problem-solving domain, with FDRs exhibiting better problem-solving performance on the NAB-Mazes task. FDRs also exhibited a trend for enhanced performance when compared to HCs. This result is novel, suggesting that FDRs may have superior planning ability, and that problem solving performance is unlikely to indicate genetic vulnerability for BD. No significant differences were found between the FDR and BD groups on the domains of verbal learning or speed of processing, failing to support our prediction that the magnitude of dysfunction in FDRs on these measures would be intermediate to that of BD patients and HCs.

There are some considerations to take into account when interpreting these findings. These largely relate to the potential insensitivity of some of the tests in the MCCB for detecting cognitive deficits that have been previously noted in BD and FDR samples. This may explain the absence of significant differences in our BD group compared to HCs on speed of processing and problem solving domains, or why the magnitude of the BD-HC effect for verbal learning was smaller than that seen in other studies that have used more 
complex measures such as the California Verbal Learning Task (Arts et al., 2008; MartínezArán et al., 2004; Torrent et al., 2006; Zubieta et al., 2001). It has been argued that although the HVLT-R may be usefully applied to BD in the context of pro-cognitive trials or for the integration of data across samples in which the MCCB has been used, it may not be particularly useful as an exploratory measure for understanding specific memory processes in BD (Van Rheenen and Rossell, 2014b). It is therefore likely that more challenging verballearning measures will be more effective in detecting the more subtle deficits that are assumed to be present in FDR samples (Burdick et al., 2011a; Yatham et al., 2010).

The MCCB also includes only one executive measure (NAB-Mazes), which to date has not been well researched in BD studies. The NAB-Mazes assesses reasoning and problem solving, and elicits smaller effects in BD than those seen for measures of set shifting and response inhibition (Bora et al., 2009; Burdick et al., 2011b; Van Rheenen and Rossell, 2014a). This suggests that not all executive functions are equally impaired in BD samples. Thus it is possible that the NAB-Mazes task may not be an adequate measure of executive function in BD or FDR samples, and that other measures such as the Wisconsin Card Sorting Test, the Stroop Colour-Word Interference Test, and the Trail Making Test-B may be more sensitive measures for these populations (Burdick et al., 2011a; Van Rheenen and Rossell, 2014a; Yatham et al., 2010). Similarly, given the simplicity of the TMT-A, and in the context of significant impairments in FDR and BD groups evidenced on the BACS-SC, it is plausible that the absence of significant impairments on this task in our study is related to an insensitivity in assessing the subtle speed of processing difficulties that are known to affect these samples, but which are revealed on more complex tasks like the BACS-SC (Antila et al., 2007b; Daban et al., 2012).

Other limitations include our small sample size. This was a factor of the inherent difficulty in recruiting from psychiatric and FDR populations, and likely reduced the power 
of our study to detect effects that may truly be present. For example, in our sample we did not find significant differences amongst groups to represent an intermediate pattern of performance in the FDR sample relative to BD patients and HCs. Further, given the variability in medications in the BD sample, the effects of psychotropic medication on cognition were not accounted for in this study. Although a limitation to all cognitive studies, research explicitly focussing on the effects of medication on cognition in BD should be prioritised. Nonetheless the absence of differences between BD and medication - free FDR patients here, in the context of significant group differences on some measures compared with HCs, suggests that medication effects were unlikely to have played a large role in the present findings.

Despite these limitations, the current work does address some shortcomings from previous research, with a major strength relating to an enhanced understanding of the importance of the age of inclusion criteria for FDRs. This criteria has varied significantly between previous studies, with the large majority having tended to include participants as young as 18 , who have arguably not surpassed the risk to go on to develop BD. To overcome this, the current study ensured all FDRs were past the modal age of BD onset of $\sim 25$ years of age. Furthermore, FDRs selected to participate in this study were unrelated to the BD sample, to avoid influences on cognitive performance that may be related to shared familial environments (Hart et al., 2007; Tucker-Drob et al., 2013). Despite the careful selection of our FDR sample, our results still largely overlapped with those of previous studies that found FDR impairments in verbal learning and processing speed. Thus it appears that regardless of whether cognitive performance in FDR individuals is measured prior to or after the modal age of $\mathrm{BD}$ onset, performance on these domains represent promising candidate endophenotypes of true familial risk for BD rather than of the potential prodromal expression of the disease itself. As the verbal learning impairments in FDRs who were unrelated to the 
BD patients in our study is consistent with recent work using sibling pairs discordant for BD (Russo et al., In press), further evidence of a genetic susceptibility for impairment on this domain may be inferred.

In sum, this study was the first to directly compare the cognitive profile of BD individuals, FDRs and HCs using the MCCB. Our data indicates that BD patients and FDRs have impaired verbal learning performance as well as selective impairments on the BACS-SC - a measure of processing speed; and the WMS-SS - a measure of spatial working memory. Since the need to hold and manipulate visual information mentally is an important component of the BACS-SC, it is not clear whether the deficits observed on the BACS-SC in our sample reflect a spatial working memory deficit, or a true underlying deficit in speed of processing. More work is required to investigate this further. 
Role of funding source

Australian Rotary Health/Bipolar Expedition, the Helen McPherson Smith Trust and an NHMRC Early Career Fellowship (to TVR).

\section{Conflict of interest}

The authors declare no conflict of interest.

\section{Acknowledgements}

The authors would like to acknowledge the financial support of the Australian Rotary Health/Bipolar Expedition, the Helen McPherson Smith Trust and the NHMRC. We also thank Mr Michael Reynolds for his assistance with data collection. 


\section{References}

Antila, M., Tuulio-Henriksson, A., Kieseppa, T., Eerola, M., Partonen, T., Lonnqvist, J., 2007a. Cognitive functioning in patients with familial bipolar I disorder and their unaffected relatives. Psychol. Med. 37, 679-687.

Antila, M., Tuulio-Henriksson, A., Kieseppa, T., Eerola, M., Partonen, T., Lonnqvist, J., 2007b. Cognitive functioning in patients with familial bipolar I disorder and their unaffected relatives. Psychological Medicine 37, 679-687.

Arts, B., Jabben, N., Krabbendam, L., Van Os, J., 2008. Meta-analyses of cognitive functioning in euthymic bipolar patients and their first-degree relatives. Psychological Medicine 38, 771-785.

Balanza-Martinez, V., Rubio, C., Selva-Vera, G., Martinez-Aran, A., Sanchez-Moreno, J., Salazar-Fraile, J., Vieta, E., Tabares-Seisdedos, R., 2008. Neurocognitive endophenotypes (endophenocognitypes) from studies of relatives of bipolar disorder subjects: A systematic review. Neuroscience and biobehavioral reviews 32, 14261438.

Baldessarini, R.J., Tondo, L., Vázquez, G.H., Undurraga, J., Bolzani, L., Yildiz, A., Khalsa, H., Mandir, K., Lai, M., Lepri, B., 2012. Age at onset versus family history and clinical outcomes in 1,665 international bipolar - I disorder patients. World Psychiatry 11, 40-46.

Bauer, I.E., Wu, M.-J., Frazier, T.W., Mwangi, B., Spiker, D., Zunta-Soares, G.B., Soares, J.C., 2016. Neurocognitive functioning in individuals with bipolar disorder and their healthy siblings: a preliminary study. J. Affect. Disord. 1, 51-56.

Bearden, C.E., Woogen, M., Glahn, D.C., 2010. Neurocognitive and neuroimaging predictors of clinical outcome in bipolar disorder. Curr Psychiatry Rep 12, 499-504.

Bora, E., Vahip, S., Akdeniz, F., Ilerisoy, H., Aldemir, E., Alkan, M., 2008. Executive and verbal working memory dysfunction in first-degree relatives of patients with bipolar disorder. Psychiatry Research 161, 318-324.

Bora, E., Yucel, M., Pantelis, C., 2009. Cognitive endophenotypes of bipolar disorder: a meta-analysis of neuropsychological deficits in euthymic patients and their firstdegree relatives. Journal of Affective Disorders 113, 1-20.

Burdick, K.E., Goldberg, T.E., Cornblatt, B.A., Keefe, R.S., Gopin, C.B., Derosse, P., Braga, R.J., Malhotra, A.K., 2011a. The MATRICS consensus cognitive battery in patients with bipolar I disorder. Neuropsychopharmacology 36, 1587-1592.

Burdick, K.E., Goldberg, T.E., Cornblatt, B.A., Keefe, R.S., Gopin, C.B., DeRosse, P., Braga, R.J., Malhotra, A.K., 2011b. The MATRICS Consensus Cognitive Battery in Patients with Bipolar I Disorder. Neuropsychopharmacology 36, 1587-1592.

Cardenas, S.A., Kassem, L., Brotman, M.A., Leibenluft, E., McMahon, F.J., 2016. Neurocognitive functioning in euthymic patients with bipolar disorder and unaffected relatives: A review of the literature. Neuroscience \& Biobehavioral Reviews 69, 193215.

Christensen, M.V., Kyvik, K.O., Kessing, L.V., 2006. Cognitive function in unaffected twins discordant for affective disorder. Psychological Medicine 36, 1119-1129.

Clark, L., Goodwin, G.M., 2004. State-and trait-related deficits in sustained attention in bipolar disorder. European archives of psychiatry and clinical neuroscience 254, 6168.

Clark, L., Kempton, M.J., Scarna, A., Grasby, P.M., Goodwin, G.M., 2005a. Sustained attention-deficit confirmed in euthymic bipolar disorder but not in first-degree 
relatives of bipolar patients or euthymic unipolar depression. Biological Psychiatry 57, 183-187.

Clark, L., Sarna, A., Goodwin, G.M., 2005b. Impairment of executive function but not memory in first-degree relatives of patients with bipolar I disorder and in euthymic patients with unipolar depression. American Journal of Psychiatry 162, 1980-1982.

Daban, C., Mathieu, F., Raust, A., Cochet, B., Scott, J., Etain, B., Leboyer, M., Bellivier, F., 2012. Is processing speed a valid cognitive endophenotype for bipolar disorder? J Affect Disord 139, 98-101.

Douglas, K.M., Van Rheenen, T.E., 2016. Current Treatment Options for Cognitive Impairment in Bipolar Disorder: a Review. Current Treatment Options in Psychiatry, $1-26$.

Ferrier, I.N., Chowdhury, R., Thompson, J.M., Watson, S., Young, A.H., 2004a. Neurocognitive function in unaffected first- degree relatives of patients with bipolar disorder: A preliminary report. Bipolar Disorders 6, 319-322.

Ferrier, I.N., Chowdhury, R., Thompson, J.M., Watson, S., Young, A.H., 2004b.

Neurocognitive function in unaffected first-degree relatives of patients with bipolar disorder: a preliminary report. Bipolar Disorders 6, 319-322.

Glahn, D.C., Bearden, C., Niendam, T.A., Escamilla, M.A., 2004. The feasibility of neuropsychological endophenotypes in the search for genes associated with bipolar affective disorder. Bipolar Disorders 6, 171-182.

Gourovitch, M.L., Torrey, E.F., Gold, J.M., Randolph, C., Weinberger, D.R., Goldberg, T.E., 1999. Neuropsychological performance of monozygotic twins discordant for bipolar disorder. Biological Psychiatry 45, 639- 119.

Hart, S.A., Petrill, S.A., Deckard, K.D., Thompson, L.A., 2007. SES and CHAOS as environmental mediators of cognitive ability: A longitudinal genetic analysis. Intelligence 35, 233-242.

Hasler, G., Drevets, W.C., Gould, T.D., Gottesman, I.I., Manji, H.K., 2006. Toward constructing an endophenotype strategy for bipolar disorders. Biological Psychiatry 60, 93-105.

Hellvin, T., Sundet, K., Simonsen, C., Aminoff, S.R., Lagerberg, T.V., Andreassen, O.A., Melle, I., 2012. Neurocognitive functioning in patients recently diagnosed with bipolar disorder. Bipolar Disorders 14, 227-238.

Joy, S., Kaplan, E., Fein, D., 2004. Speed and memory in the WAIS-III Digit Symbol-Coding subtest across the adult lifespan. Arch Clin Neuropsychol 19, 759-767.

Kieseppä, T., Tuulio-Henriksson, A., Haukka, J., Van Erp, T., Glahn, D., Cannon, T.D., Partonen, T., Kaprio, J., Lönnqvist, J., 2005. Memory and verbal learning functions in twins with bipolar-I disorder, and the role of information-processing speed. Psychological Medicine 35, 205-215.

Klimes-Dougan, B., Ronsaville, D., Wiggs, E.A., Martinez, P.E., 2006. Neuropsychological functioning in adolescent children of mothers with a history of bipolar or major depressive disorders. Biological Psychiatry 60, 957-965.

Kremen, W.S., Faraone, S.V., Seidman, L.J., Pepple, J.R., Tsuang, M.T., 1998. Neuropsychological risk indicators for schizophrenia: a preliminary study of female relatives of schizophrenic and bipolar probands. Psychiatry Research 79, 227-240.

Kulkarni, S., Jain, S., Janardhan Reddy, Y.C., Kumar, K.J., Kandavel, T., 2010. Impairment of verbal learning and memory and executive function in unaffected siblings of probands with bipolar disorder. Bipolar Disorders 12, 647-656.

Kurtz, M.M., Gerraty, R.T., 2009. A meta-analytic investigation of neurocognitive deficits in bipolar illness: profile and effects of clinical state. Neuropsychology 23, 551-562. 
Lezak, M.D., Howieson, D.B., Loring, D.W., Hannay, H.J., Fischer, J.S., 2004.

Neuropsychological assessment.

Mann-Wrobel, M.C., Carreno, J.T., Dickinson, D., 2011. Meta-analysis of neuropsychological functioning in euthymic bipolar disorder: an update and investigation of moderator variables. Bipolar Disorders 13, 334-342.

Martinez-Aran, A., Vieta, E., Colom, F., Reinares, M., Benabarre, A., Gasto, C., Salamero, M., 2000. Cognitive dysfunctions in Bipolar Disorder: Evidence of neuropsychological disturbaces. Psychotherapy and Psychosomatics 69, 2-18.

Martinez-Aran, A., Vieta, E., Colom, F., Torrent, C., Sanchez-Moreno, J., Reinares, M., Benabarre, A., Goikolea, J.M., Brugue, E., Daban, C., Salamero, M., 2004. Cognitive impairment in euthymic bipolar patients: Implications for clinical and functional outcome. Bipolar Disorders 6, 224-232.

Martínez-Arán, A., Vieta, E., Reinares, M., Colom, F., Torrent, C., Sánchez-Moreno, J., Benabarre, A., Goikolea, J.M., Comes, M., Salamero, M., 2004. Cognitive function across manic or hypomanic, depressed, and euthymic states in bipolar disorder. American Journal of Psychiatry 161, 262-270.

McIntosh, A.M., Harrison, L.K., Forrester, K., Lawrie, S.M., Johnstone, E.C., 2005. Neuropsychological impairments in people with schizophrenia or bipolar disorder and their unaffected relatives. British Journal of Psychiatry 186, 378-385.

Montgomery, S.A., Asberg, M., 1979. A new depression scale designed to be sensitive to change. British Journal of Psychiatry 134, 382-389.

Nehra, R., Chakrabarti, S., Pradhan, B.K., Khehra, N., 2006. Comparison of cognitive functions between first- and multi-episode bipolar affective disorders. J. Affect. Disord. 93, 185-192.

Nuechterlein, K., Green, M.F., 2006. MATRICS Consensus Cognitive Battery manual. MATRICS Assessment Inc., USA.

Pierson, A., Jouvent, R., Quintin, P., Perez-Diaz, F., Leboyer, M., 2000. Information processing deficits in relatives of manic depressive patients. Psychological Medicine 30, 545-555.

Robinson, L.J., Ferrier, I.N., 2006. Evolution of cognitive impairment in bipolar disorder: a systematic review of cross-sectional evidence. Bipolar Disorders 8, 103-116.

Robinson, L.J., Thompson, J.M., Gallagher, P., Goswami, U., Young, A.H., Ferrier, I.N., Moore, P.B., 2006. A meta-analysis of cognitive deficits in euthymic patients with bipolar disorder. Journal of Affective Disorders 93, 105-115.

Russo, M., Van Rheenen T.E, Shanahan M, Mahon K, Mercedes Perez-Rodriguez M, Cuesta-Diaz, A., AK, M., Burdick, K., In press. Neurocognitive subtypes in patients with bipolar disorder and their unaffected siblings.

Sheehan, D.V., Lecrubier, Y., Sheehan, K.H., Amorim, P., Janavs, J., Weiller, E., Hergueta, T., Baker, R., Dunbar, G.C., 1998. The Mini-International Neuropsychiatric Interview (MINI): The development and validation of a structured diagnostic psychiatric interview for DSM-IV and ICD-10. Journal of Clinical Psychiatry 59, 22-33.

Sobczak, S., Honig, A., Schmitt, J.A., Riedel, W.J., 2003. Effects of acute tryptophan depletion on mood and cortisol release in first-degree relatives of type I and type II bipolar patients and healthy matched controls. Neuropsychopharmacology 27, 834842.

Strauss, E., Sherman, E.M., Spreen, O., 2006. A compendium of neuropsychological tests: Administration, norms, and commentary. Oxford University Press, USA.

Thompson, J.M., Gallagher, P., Hughes, J.H., Watson, S., Gray, J.M., Ferrier, I.N., Young, A.H., 2005. Neurocognitive impairment in euthymic patients with bipolar affective disorder. British Journal of Psychiatry 186, 32-40. 
Tombaugh, T.N., Kozak, J., Rees, L., 1999. Normative Data Stratified by Age and Education for Two Measures of Verbal Fluency: FAS and Animal Naming. Archives of Clinical Neuropsychology 14, 167-177.

Torrent, C., Martínez-Arán, A., Daban, C., Sánchez-Moreno, J., Comes, M., Goikolea, J.M., Salamero, M., Vieta, E., 2006. Cognitive impairment in bipolar II disorder. British Journal of Psychiatry 189, 254-259.

Torres, I.J., Boudreau, V.G., Yatham, L.N., 2007. Neuropsychological functioning in euthymic bipolar disorder: A meta-analysis. Acta Psychiatrica Scandinavica 116, 17 26.

Trivedi, J.K., Goel, D., Dhyani, M., Sharma, S., Singh, A.P., Sinha, P.K., Tandon, R., 2008. Neurocognition in first - degree healthy relatives (siblings) of bipolar affective disorder patients. Psychiatry Clin. Neurosci. 62, 190-196.

Tucker-Drob, E.M., Briley, D.A., Harden, K.P., 2013. Genetic and Environmental Influences on Cognition Across Development and Context. Curr Dir Psychol Sci 22, 349-355.

Van Rheenen, T., Lewandowski, K., Tan, E., Ospina, L., Ongur, D., Neill, E., Gurvich, C., Pantelis, C., Malhotra, A., Rossell, S., 2017. Characterizing cognitive heterogeneity on the schizophrenia-bipolar disorder spectrum. Psychol. Med., 1-17.

Van Rheenen, T.E., Bryce, S., Tan, E.J., Neill, E., Gurvich, C., Louise, S., Rossell, S.L., 2016. Does cognitive performance map to categorical diagnoses of schizophrenia, schizoaffective disorder and bipolar disorder? A discriminant functions analysis. J. Affect. Disord. 192, 109-115.

Van Rheenen, T.E., Meyer, D., Rossell, S.L., 2014. Pathways between neurocognition, social cognition and emotion regulation in bipolar disorder. Acta Psychiatrica Scandinavica 130, 397-405.

Van Rheenen, T.E., Rossell, S.L., 2014a. An Empirical Evaluation of the MATRICS Consensus Cognitive Battery in Bipolar Disorder. Bipolar Disorders 16, 318-325.

Van Rheenen, T.E., Rossell, S.L., 2014b. An investigation of the component processes involved in verbal declarative memory function in bipolar disorder; utility of the Hopkins Verbal Learning Test-Revised. J. Int. Neuropsychol. Soc. 20, 1-9.

Wechsler, D., 2001. Wechsler Test of Adult Reading: WTAR. Psychological Corporation. Yatham, L.N., Torres, I.J., Malhi, G.S., Frangou, S., Glahn, D.C., Bearden, C.E., Burdick, K.E., Martínez - Arán, A., Dittmann, S., Goldberg, J.F., 2010. The International Society for Bipolar Disorders-Battery for Assessment of Neurocognition (ISBD BANC). Bipolar disorders 12, 351-363.

Young, R., Biggs, J., Ziegler, V., Meyer, D., 1978. A rating scale for mania: Reliability, validity and sensitivity. British Journal of Psychiatry 133, 429-435.

Zalla, T., Joyce, C., Szöke, A., Schürhoff, F., Pillon, B., Komano, O., Perez-Diaz, F., Bellivier, F., Alter, C., Dubois, B., Rouillon, F., Houde, O., Leboyer, M., 2004. Executive dysfunctions as potential markers of familial vulnerability to bipolar disorder and schizophrenia. Psychiatry Research 121, 207-217.

Zubieta, J.-K., Huguelet, P., O'Neil, R.L., Giordani, B.J., 2001. Cognitive function in euthymic bipolar I disorder. Psychiatry research 102, 9-20. 